\title{
Nutrição em lactentes de um bairro de Manaus, Amazonas $\left(^{*}\right)$
}

\author{
Roger Shrimpton $(* *$ j \\ Rodolfo Giugliano ${ }^{*{ }^{*} *}$ )
}

\begin{abstract}
Resumo
O estado nutricional, padrōes alimentares, infecções intestinais e respiratórias foram verificados em 82 crianças no primeiro ano de vida de uma populaçāo urbana de Manaus, Amazonas, nos meses de maio e junho de 1976. Alto índice de desnutrição acompanhado de alta morbilidade foi encontrado. Constatou-se também alta taxa de recém-nascidos de baixo peso, sugerindo uma freqüência elevada de desnutrição intra-útero. Os fatores predisponentes mais importantes foram atribuícos à substituição precoce da amamentação pelo leite artificial, à falta de orientação ou orientação errônea das mães compreendendo a ausência total de uma puericultura dirigida às condições sócio-econômicas da região.
\end{abstract}

\section{INTRODUÇÃo}

A Bacia Amazônica corresponde a $47 \%$ da superfície do Brasil, contendo apenas $4 \%$ da população brasileira. Essa população se distribui em $52 \%$ na área urbana e $48 \%$ na área rural (Anuário Estatístico do Brasil, 1975). $\mathrm{O}$ estado nutricional dessa população foi pouco estudado.

Castro (1968) conclui em seus estudos sobre indígenas que "quando mais se aproxima do Baixo Amazonas (região ribeirinha) menor é a capacidade física do indivíduo, e tanto maior quando voltamos para o Alto e Médio Amazonas. Parece até que há uma inversão entre o primitivismo e a civilização". Chaves (1972) cita na "Introdução à Geografia Médica do Brasil" que "realmente a civilização, muitas vezes, concorre para a piora da situação alimentar das populações. O desmame precoce, que é uma das principais causas da desnutrição grave e que resulta de diversos fatores, não ocorre entre os nativos".
Provavelmente, o único estudo de alimentação infantil no Amazonas (Comissão Nacional de Alimentação, 1959) foi realizado nos anos de 1954 à 1956, incluindo inquéritos entre 538 mulheres, em diversas cidades da $\mathrm{Ba}-$ cia Amazônica e concluiu, quanto à amamentação, que $51 \%$ das mäes amamentavam seus filhos de 12 até 36 meses; somente $10 \%$ deixavam de amamentar entre os $3 .^{\circ}$ e o $6 .^{\circ}$ mês e $7 \%$ cessavam a amamentação até o $3 .^{\circ}$ mês (Shrimpton, 1975).

A cidade de Manaus, localizada no Médio Amazonas contém uma população de aproximadamente 600.000 habitantes que represen$\operatorname{tam} 25 \%$ da população urbana da Bacia Amazônica (Anuário Estatístico do Brasil, 1975). Nos últimos 10 anos, Manaus sofreu um crescimento rápido e considerável.

O objetivo deste trabalho foi estudar os padrões alimentares e estado nutricional no primeiro ano de vida de uma populaçăo urbana da Bacia Amazônica.

\section{MATERIAIS E MÉTODOS}

Foi escolhido o bairro Coroado, Manaus, com uma população de aproximadamente 15.000 habitantes (') distante $12 \mathrm{~km}$ do centro da cidade. A populaçăo é de baixo nível sócio-econômico, provavelmente representativo de pelo menos $30 \%$ da populaçăo manauense baseado em tipo de habitação e condiçōes sanitárias (CODEAMA, SEPLAN, 1974). O bairro compõe-se quase exclusivamente de casas de madeira e não dispõe de rede de água, luz ou esgoto.

Apoiados na estrutura populacional brasileira (Anuário Estatístico do Brasil, 1975) estimamos em 600 o número de crianças no pri-

\footnotetext{
(*) F- Financiado pelo Conselho Nacional de Desenvolvimento Científico e Tecnológico (CNPq).

("*) - Instituto Nacional de Pesquisas da Amazônia, Manaus.

(*) - Instituto Nacional de Pesquisas da Amazônia e Hospital de Moléstias Tropicais, Manaus.

( 1 ) - Baseados em dados fornecidos pela Superintendência das Campanhas de Saúde Pública (SUCAM).
} 
meiro ano de vida. Oitenta e duas crianças foram examinadas, representando $13 \%$ da população em estudo. Através de um mapa do bairro (") foram selecionadas, por sorteio, quadras a serem visitadas. Todas as casas dessas quadras foram percorridas e as crianças no primeiro ano de vida examinadas.

A pesquisa foi realizada nos meses de maio e junho de 1976, correspondente ao fim da época chuvosa e o início da época seca.

Em cada criança foram realizados estudos clínico, antropométrico e parasitológico de fezes. A mãe ou acompanhante foi entrevistada sobre condições de parto e nascimento, antecedentes patológicos, conceitos de alimentação infantil e a alimentação da criança.

\section{Resultados}

\section{I - CARACTERÍSTICAS DA POPULAÇÃO ESTUDADA}

Das famílias estudadas $40 \%$ das mães e $38 \%$ dos pais nasceram em Manaus, enquanto $43 \%$ das mães e $33 \%$ dos pais vieram do interior do Estado do Amazonas. O restante é proveniente de outros estados, principalmente do Pará. Setenta e cinco por cento dessas famílias eram integradas, ou seja, pai e mãe vivendo juntos. Somente $15 \%$ das mães tra- balhavam fora e $7 \%$ dos pais estavam desempregados.

A fonte de água é constituída por cacimba ou poço individual em $21 \%$ das casas visitadas e coletivo no restante. A água é utilizada em sua maioria "in natura"; fervida previamente em $12 \%$ e fervida somente para o bebê em $37 \%$ das crianças estudadas. Quarenta e oito por cento referiam ser a casa própria, porém devemos ressaltar que o bairro se originou de invasão de terras, estando sua condição ainda por ser legalizada.

Das crianças examinadas $51 \%$ eram do sexo masculino e $49 \%$ do sexo feminino. Sua distribuiçăo por trimestre de vida correspondeu a $29(35,0 \%)$ no $1 .^{\circ}$ trimestre, $19(23,2 \%)$ no $2 .^{\circ}, 26(31,7 \%)$ no $3 .^{\circ}$ e $8(9,8 \%)$ no $4 .^{\circ}$ trimestre.

\section{II - PADRÕES ALIMENTARES}

Das crianças nos primeiros três meses de vida, somente $2(6,9 \%)$ estavam recebendo exclusivamente leite materno. O método mais comum de alimentação é o aleitamento misto. correspondendo a $20(69,0 \%)$ das crianças até o $3 .^{\circ}$ mês de vida. A Tabela I mostra o número de crianças recebendo leite materno, leite artificial ou ambos nos respectivos trimestres do primeiro ano de vida.

\section{TABELA I}

Tipo de aleitamento por trimestre no primeiro ano de vida em um bairro de Manaus, 1976

\begin{tabular}{|c|c|c|c|c|c|c|c|c|c|c|}
\hline \multirow{3}{*}{$\begin{array}{c}\text { TIPO } \\
\text { DE } \\
\text { LEITE }\end{array}$} & \multicolumn{8}{|c|}{ idade das crianças em meses } & \multirow{2}{*}{\multicolumn{2}{|c|}{ TOTAL }} \\
\hline & \multicolumn{2}{|c|}{$0-2$} & \multicolumn{2}{|c|}{$3-5$} & \multicolumn{2}{|c|}{$6-8$} & \multicolumn{2}{|c|}{$9-11$} & & \\
\hline & n. ${ }^{\circ}$ & $\%$ & n. ${ }^{\circ}$ & $\%$ & n. ${ }^{\circ}$ & $\%$ & $\mathrm{n} .^{\circ}$ & $\%$ & n. ${ }^{\circ}$ & $\%$ \\
\hline Artificial & 07 & 24,1 & 05 & 26,3 & 13 & 50,0 & 06 & 75,0 & 31 & 37,8 \\
\hline Materno & 02 & 6,9 & 01 & 5,3 & 02 & 7,7 & 01 & 12,5 & 06 & 7,3 \\
\hline Misto & 20 & 69,0 & 13 & 68,4 & 11 & 42,3 & 01 & 12,5 & 45 & 54,9 \\
\hline TOTAL & 29 & 100,0 & 19 & 100,0 & 26 & 100,0 & 08 & 100,0 & 82 & 100,0 \\
\hline
\end{tabular}

(*) - Baseados em dados fornecidos pela Superintendência das Campanhas de Saúde Pública (SUCAM). 
Os resultados evidenciam que o leite materno está numa proporção constantemente baixa em cada trimestre, ao passo que com o tempo, o aleitamento misto é substituído pelo artificial.

Setenta e seis crianças $(92,7 \%)$ estavam recebendo mamadeira, sendo a maioria delas feitas com leite "engrossado" com farináceos e açúcar (vide Tabela II). Somente 7 crianças $(8,5 \%)$ tomavam apenas leite enquanto duas recebiam apenas farináceos. Das 74 crianças $(90,2 \%)$ recebendo leite, $41(55,4 \%)$ recebiam preparados de leite em pó modificado e 31 $(41,9 \%)$ leite em pó integral. Duas crianças $(2,4 \%)$ eram alimentadas com leite condensado. No que se refere ao enriquecimento usado nas mamadeiras, açúcar era usado para 61 crianças $(80,3 \%)$, farinha de mandioca para $40(52,6 \%)$ e maizena para $7(9,2 \%)$. O enriquecimento mais comum era uma combinação de farinha de mandioca e açúcar, encontrado em 32 casos $(43,2 \%)$.

\section{TABELA II}

Composição da dieta de crianças com alimentação artificial e mista em um bairro de Manaus, 1976

\begin{tabular}{lcc}
\hline \multicolumn{2}{c}{ N.॰ de casos } & Porcentagem \\
\hline A. Tipos de leites usados: & & \\
1. pó modificado & 41 & 55,4 \\
2. pó integral & 31 & 41,9 \\
3. condensado & 02 & 2,4 \\
B. Freqüência dos & & \\
enriquecimentos: & & \\
1. açúcar & 61 & 80,3 \\
2. farinha de mandioca & 40 & 52,6 \\
3. Maizena & 07 & 9,2 \\
4. mucilon & 06 & 8,7 \\
5. outros & 03 & 3,9 \\
\hline
\end{tabular}

Com relação à introdução de alimentos da dieta adulta, apenas 5 mães $(6,1 \%)$ revelaram que já os davam às crianças. A média etária das cinco crianças era de 8.8 meses.

Quando as mães foram questionadas sobre qual seria o melhor alimento para a criança nos primeiros seis meses de vida 15 $(18,3 \%)$ responderam leite materno, $29(35,4 \%)$ leite artificial ou misto e $25(30,5 \%)$ não souberam responder. Em 13 casos $(15,8 \%)$ a pergunta não foi efetuada por motivos diversos.
Somente 14 das mäes $(17,1 \%)$ admitiram terem sido orientadas quanto à alimentaçăo de seus filhos. Em sete casos, por médicos e no restante por enfermeiras, parteiras ou parentes próximos. Apenas uma das mães foi orientada no sentido de que o leite materno seria o melhor alimento.

\section{III - MORBILIDADE}

A presença de diarréia, sintomas respiratórios (coriza, tosse) ou febre no momento do exame foi verificada. Perguntamos também sobre os quadros diarréicos ou respiratórios apresentados anteriormente pela criança. Os resultados são apresentados nas Tabelas III e IV.

TABELA III

Freqüência de diarréia, sintomas respiratórios ou febre no momento do exame em crianças no primeiro ano de vida em um bairro de Manaus, 1976

\begin{tabular}{|c|c|c|c|c|c|c|}
\hline & \multicolumn{6}{|c|}{ idade em meses } \\
\hline & \multicolumn{2}{|c|}{$0-2$} & \multicolumn{2}{|c|}{$3-5$} & \multicolumn{2}{|c|}{$6-11$} \\
\hline & n. ${ }^{a}$ & $\%$ & $\mathrm{n}{ }^{a}$ & $\%$ & n. ${ }^{\circ}$ & $\%$ \\
\hline Diarréia & 08 & 27,6 & 07 & 36,8 & 12 & 35,5 \\
\hline S. Resp. & 08 & 27,6 & 10 & 52,6 & 18 & 529 \\
\hline Febre & 01 & 3,4 & 04 & 21,0 & 08 & 26,5 \\
\hline
\end{tabular}

\section{TABELA IV}

Freqüência de antecedentes de diarréia, sintomas respiratórios ou febre em crianças no primeiro ano de vida em um bairro de Manaus, 1976

\begin{tabular}{|c|c|c|c|c|c|c|}
\hline & \multicolumn{6}{|c|}{ idade em meses } \\
\hline & \multicolumn{2}{|c|}{$0-2$} & \multicolumn{2}{|c|}{$3-5$} & \multicolumn{2}{|c|}{$6-11$} \\
\hline & n. ${ }^{\circ}$ & $\%$ & n. ${ }^{\circ}$ & $\%$ & n. & $\%$ \\
\hline Diarréia & 15 & 55,5 & 12 & 80,0 & 22 & 62,7 \\
\hline S. Resp. & 10 & 37,0 & 07 & 46,7 & 26 & 76,8 \\
\hline Febre & 08 & 29,6 & 05 & 33,3 & 26 & 76,8 \\
\hline
\end{tabular}


As crianças foram divididas quanto à faixa etária nos primeiros trimestres e no segundo semestre com o objetivo de verificarmos a precocidade com que são submetidas às infecções intestinais e respiratórias. Assim, já no primeiro trimestre, 8 crianças $(27,6 \%)$ apresentavam diarréia no moniento do exame e 15 crianças $(55,5 \%)$ já haviam sofrido quadros diarreicos anteriores.

O exame parasitológico de fezes foi realizado em $72(87,8 \%)$ das crianças examinadas. Os resultados estão colocados na Tabela V.

\section{TABELA V}

Parasitológico de fezes em crianças no primeiro ano de vida em um bairro de Manaus, 1976

\begin{tabular}{ccc}
\hline TIPO & N. & $\%$ \\
\hline Giardia lamblia & 07 & 8,3 \\
Ascaris lumbricoides & 02 & 2,8 \\
Ancilostoma sp. & 01 & 1,4 \\
\hline
\end{tabular}

\section{IV - ESTADO NUTRICIONAL}

O estado nutricional foi avaliado através da classificação de Gomez et al., (1956) e os resultados são mostrados na Tabela VI.

\section{TABELA VI}

Avaliação do estado nutricional através de Gomez em crianças no primeiro ano de vida em um bairro de Manaus, 1976

\begin{tabular}{ccc}
\hline GRAUS DE GOMEZ & N.॰ & $\%$ \\
\hline Normal & 49 & 64,5 \\
1 & 20 & 26,3 \\
2 & 07 & 9,2 \\
3 & 0 & 0 \\
\hline
\end{tabular}

O estado nutricional foi também verifica. do através da classificação sugerida por Waterlow (1972), que evidencia os efeitos do ambiente sobre altura e sobre peso, como é expresso pela Tabela VII.
Em relação ao peso por idade e estatura por idade, somente 76 das 82 crianças puderam ser computadas, em virtude da perda dos dados em 2 casos e de em 4 casos as crianças se apresentarem com peso inferior a $2,5 \mathrm{~kg}$ ou estatura abaixo de $48 \mathrm{~cm}$, sendo então consideradas recém-nascidas de baixo peso. Essas 4 crianças eram da amostra do primeiro mês e representam $36,4 \%$ dessa faixa etária.

\section{TABELA VII}

Avaliação do estado nutricional através de Waterlow em crianças no primeiro ano de vida em um bairro de Manaus, 1976

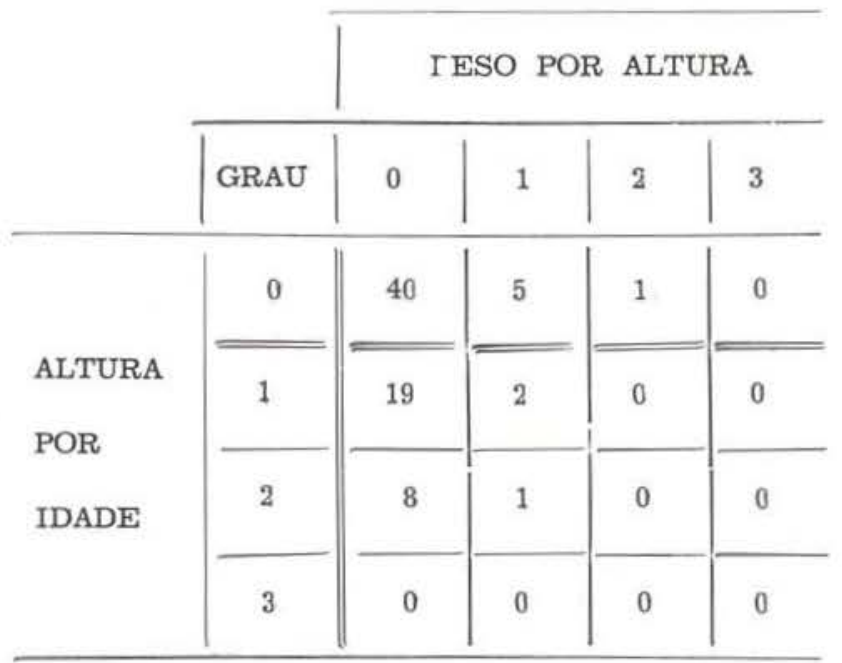

Foi anotado também o peso de nascimento das crianças em estudo e considerados para análise os casos nos quais havia comprovante de maternidade. Nos 35 casos em que esses critérios foram observados, $6 \quad(17,1 \%)$ nasceram com peso inferior a $2,5 \mathrm{~kg}$. A média do peso de nascimento era de $3,1 \mathrm{~kg} \pm 1 \mathrm{~kg}$ e da altura $48,3 \mathrm{~cm} \pm 5,8 \mathrm{~cm}$ ( \pm 2 D.P.).

\section{DISCUSSÃo}

A classificação nutricional de Gomez (1956), a mais antiga e conhecida classificação quanto à severidade da má nutrição, é baseada nos deficits de peso por idade em relação ao $50^{\circ}$ percentil do Padrão de Harvard (Stuart \& Stevenson, 1959). Os limites de cada grau de Gomez são baseados em observações clínicas em crianças no México e não possuem bases estatísticas diretas. De acordo com a 
classificação de Gomez, $35,5 \%$ das crianças es tudadas no primeiro ano de vida estavam sotrendo de má nutrição.

A classificação de Waterlow (1974) permite uma diferenciação da distorção do crescimento com relação a ambos altura por idade e peso por altura. O primeiro mostra a extensão do nanismo nutricional (do Inglês "stunting") e o segundo a atrofia nutricional (do Inglês "wasting"). Ela está relacionada também ao Padrão de Harvard, sendo o limite de $\mathrm{O}$ (normalidade) correspondente a 2 desvios padrões do Padrão de Harvard, ou o $3 .^{\circ}$ percentil. Esta classificação demonstra que $7,9 \%$ da população estudada sofria de atrofia nutricional, e isto sugere que estavam sofrendo de má nutrição aguda. Outros 3,9\% tinham nanismo e atrofia nutricional enquanto $35,5 \%$ das criancas estudadas tinham apenas nanismo nutricional.

Este nanismo indica má nutrição crônica. Para a má nutrição ser crônica no primeiro ano de vida, existiu um severo retardo no crescimento intrauterino. Isto é substanciado pelo grande número de baixos pesos de nascimento. Apenas $50 \%$ das crianças nasceram em hospitais e daquelas com pesos de nascimento registrados, $17,1 \%$ eram inferiores a $2,5 \mathrm{~kg}$. Além disso, das 11 crianças examinadas no primeiro mês de vida, 4 tinham peso inferior a $2,5 \mathrm{~kg}$. Baixos pesos de nascimento são reconhecidamente causados por efeitos mais ambientais do que genéticos ou raciais e, não sendo associados a um período reduzido de gestação, refletem um ritmo retardado de crescimento intrauterino (Foetal Nutrition, 1970), que é em grande parte dependente do estado nutricional da mãe. Um grupo de pesquisadores guatemaltecos demonstrou que uma suplementação à dieta materna afeta criticamente o peso de nascimento da criança (Nut. Rev., 1973). O início rápido de um programa de suplementação dietética da mãe gestante é medida prioritária.

Para alguns pode parecer que o relacionamento entre o crescimento infantil na Bacia Amazônica e o crescimento de uma população americana não seja realista, mas já foi demonstrado que as influências genéticas nos padrōes de crescimento são pequenas em comparação com as influências ambientais (Habicht et al., 1974). A maioria dos estudos de crescimento em pré-escolares de diferentes origens étnicas, inclusive na América Central, demonstram uma grande aproximaçăo ao Padrão de Crescimento Longitudinal de Harvard (Stuart \& Stevenson, 1959).

Estudos no Peru, sobre populações indígenas mistas similares, demonstram que padrões de crescimento iguais aos de Harvard podem ser mantidos, ficando as crianças no hospital. Mas, ao retornarem a seus lares, logo demonstram um decréscimo na taxa de ciescimento (Graham \& Adrianzen, 1971).

A importância dada aos deficits de crescimento aumenta à medida que se conhece cada vez melhor a correlação entre a altura e o peso de nascimento com o desenvolvimento intelectual (Martin, 1973). Habilidades intelectuais retardadas são encontradas no menor irmão gêmeo, quando existe uma discrepância entre os pesos de nascimento (Babson et al., 1964). No Homem, a maior parte do desenvolvimento cerebral ocorre nos últimos 3 meses de vida intrauterina e nos primeiros 2 anos (Dobbing, 1974). Deficits de crescimento durante estas fases em animais demonstram deficiências na composição e na bioquímica do cérebro (Winick \& Noble, 1966), além de afetar o comportamento, podendo estas deficiências serem transmitidas a sucessivas geraçöes, apesar da dieta adequada (Chow \& Lee, 1964).

Estudos na Guatemala demonstraram os efeitos prejudiciais da diarréia sobre o processo de crescimento (Martorel et al., 1975) e enfatizaram a importância da má nutriçāo secundária causada por infecção primária. As taxas de infecção na população estudada em Manaus, além de muito altas, refletem o ambiente agressivo no qual crescem as crianças. Ampliação e orientação da rede de postos de saúde dirigidos e objetivando primordialmente a prevenção e puericultura devem estabelecer-se. O fornecimento periódico com as devidas instruções a cada família de um pequeno frasco contendo cloro para ser usado na água, verdura e legumes que se ingerem frescos, é medida prática e comprovadamente salutar. 
Indubitavelmente, o maior contribuinte a esta alta morbilidade no primeiro ano de vida é a preponderância da introdução precoce da alimentação artificial (mamadeira) e a baixa freqüência da amamentação. Os efeitos desastrosos da alimentação artificial em populações de baixo nível sócio-econômico no Brasil e em países em desenvolvimento estão bem documentados (Berg, 1973; Jelliffe, 1966; Shrimpton, 1975; Puffer \& Serrano, 1973; Waterlow, 1956). O grande valor do leite materno e da amamentação prolongada em tais populações é também conhecido de sobra (EI Valor Incomparable de la Leche Materna, 1972; Present Day Practice in Infant Feeding, 1974). Assim, o estímulo, a propagação e a recolocação da amamentação como a melhor e única fonte de alimento para as crianças até o 6 . $^{\circ}$ mês, impõe-se nessas populações.

A falta de conceito das mães entrevistadas sobre a maneira de alimentar seus filhos é composta pela não identificação do leite materno como "alimento". O conceito geral do leite materno, entretanto, é aquele de ser "fraco" e incapaz de sustentar o crescimento normal. O objetivo para a maiorı das mães parece ser a obesidade, com uma grande confusão entre uma criança "forte" e "gorda". Até mesmo o estudo da Comissão Nacional de Alimentação nos anos de 1954 a 1956, apesar da amamentação ser prolongada até o $2^{\circ}$ ano de vida, $90 \%$ das mães davam outros alimentos às crianças, durante o período de amamentação, com $24 \%$ iniciando no $1 .^{\circ}$ mês e $70 \%$ durante os primeiros 6 meses de vida. A razão para a suplementação do leite foi dada como "má saúde" ou conselho médico em mais de $50 \%$ dos casos (Shrimpton, 1975), enquanto nós sabemos que em populações sem alternativa, mesmo sendo pouco alimentadas conseguem amamentar bem e que o maior inibidor da amamentação é o fator psíquico.

A orientação da mãe que sai da maternidade de forma clara e ao nível de seu entendimento, por pessoal médico ou paramédico, são básicos para o futuro da criança. Não adianta dar "folhetos" para a mãe ler em casa como foi freqüentemente encontrado em nosso inquérito, já que $43 \%$ das mesmas eram analfabetas. Para que isso ocorra é necessário que o Setor de Nutrição da Universidade se desenvolva e se dirija objetivamente para os problemas e recursos locais servindo de base para orientação do pessoal médico e paramédico, pois as poucas mães que foram orientadas, o foram incorretamente.

\section{CONCLUSÕES}

Da população lactente estudada, $50,0 \%$ tinha deficits de crescimento em relação aos considerados limites de normalidade, demonstrando os ásperos efeitos de um ambiente pobre sobre o processo de crescimento e desenvolvimento. A alta incidência de crianças com baixo peso de nascimento sugere que a dieta da mãe gestante é inadequada. A natureza da deficiência permanece por ser estabelecida, mas a experiência de outras partes do Brasil e do mundo mostra que em tais populações a deficiência é mais de natureza quantitativa do que qualitativa (Shrimpton, 1975; Waterlow, 1975). A alta taxa de morbilidade neonatal associada com a falta de crescimento neste período da vida, é conhecida como sendo prejudicial ao desenvolvimento total do potencial intelectual genético. A tendência à redução da amamentação, conduzindo à alta morbilidade nos primeiros meses de vida é contrária aos melhores interesses da população estudada. Das poucas mães que tinham recebido conselho médico sobre como alimentar suas crianças, receberam o conselho errado.

\section{AGRADECIMENTOS}

- Maria de Fátima Silva Pinheiro pela realização dos exames parasitológicos.

- Secretaria de Saúde do Estado do Amazonas, pelo fornecimento de medicamentos que foram utilizados durante o inquérito.

\section{Summary}

In the months of May and June 1976, a study was made of the feeding patterns, nutritional and health status of 82 children in the first year of life in Manaus, Amazonas. Almost half of the children were considered to be suffering differing degrees of malnutrition accompanied by high indices of infection. A high proportion of the children had low birth weights, suggesting significant levels of 
intrauterine growth retardation and maternal malnutrition. Contributory factors were considered to be early substitution of breast milk for artificial milk, a lack of mother and child health care services and nutritional orientation. Of the few mothers who received nutritional advice, the majority received advice incompatible with their socio-economic and educational status.

\section{BIBLIOGRAFIA CITADA}

ANUÁRIO ESTATÍSTICO DO BRASIL

1975 - Apud: Instituto Brasileiro de Geografia e Estatística, Rio de Janeiro.

Babson, S.G.; Kangas, J.; Young, N. \& Bramhall, J.L. 1964 - Growth and development of twins of disimilar sizes at birth. Pediatrics, $33: 327$.

BERG, A

1973 - The Nutrition Factor. The Brookings Institution, Washington, D.C. 290 p.

BOLETIM DA COMISSÃo NACIONAL DE ALIMENTAÇÃo

1959 - Apud: Ano IV, N.o 2, Julho-Setembro. Rio de Janeiro.

Castro, G.B.

1968 - In: Simpósio Brasileiro de Alimentação e Nutrição, Resumo dos Trabalhos Apresentados, Universidade Federal de Pernambuco, Recife.

Chaves, N

1972 - In: Introdução à Geografia Médica do Brasil, eds. Lacaz, Baruzzi e Siqueira, pp. $524-528.568 \mathrm{p}$.

CHOW, B.F. \& LEE, C.J.

1964 - Effect of dietary restriction of pregnant rats on body weight gain of the offspring. J. Nutr., 82:10-18.

DOBBING, J.

1974 - The later growth of the brain and its vulnerability. Pediatrics, $53: 2-3$.

El VALOR INCOMPARABLE DE LA LECHE MATERNA

1972 - Apud: Publicación n. ${ }^{\circ}$ 250, Organización Panamericana de la Salud, Washington, D.C. $68 \mathrm{p}$.

EsTUdO ESPECÍFICO N.ㅇ 41

1974 - Apud: CODEAMA, SEPLAN, Governo do Estado do Amazonas, Manaus.

FOETAL NUTRITION

1970 - Apud: Bulletin of the World Health Organization, $43: 785$.

Graham, G.G. \& Adrianzen, B.

1971 - Growth, inheritance and environment. Paed. Res., 5 : 691-697.
Gomez, F.J.; Ramos Galvín, R.; Frenk, S.; Cravioto, J.; ChÁVEz, R. \& VÁsQuez, J.

1956 - Mortality in second and third degree malnutrition. J. Trop. Pediat., 2 : 77-83.

Habicht, J.P.; Martorell, R.; Yarbrough, C.; Malina, R.M. \& KLEIN, R.E

1974 - Height and weight standards for pre-school children - how relevant are ethnic differences in growth potential. The Lancet, p. 611, April 6

JELLIFFE, D.B.

1966 - The assessment of the nutritional status of the community. Monograph Series, 53, World Health Organization, Geneva. $271 \mathrm{p}$.

MARTIN, H.P.

1973 - Nutrition: itsrelationship to childrenis physical, mental and emotional development. Am. J. Clin. Nutr. 26:766-775.

Martorell, R.; Habicht, J.P.; Yarbrough, C.; Lechtig, A.; KLein, R.E. \& Western, K.A.

1975 - Acute morbidity and physical growth in rural Guatemalan children. J. Dis. Child., 129(11) : 1296-1301.

NUTRITION REVIEWS

1973 - Apud: 31(2) : 45-47.

PRESENT DAY PRACTICE IN INFANT FEEDING

1974 - Apud: Report on Health and Social Subjects, n. ${ }^{\circ}$ 9, H.M.S.O., London. 34 p.

SHRIMPTON, R.

1975 - Nutrition and Development in Brazil. Report for the degree of Master of Science, University of London. $134 \mathrm{p}$.

Stuart, H.C. \& STEVenson, S.S.

1959 - In: Textbook of Paediatrics, Ed. W.E. Nelson, Philadelphia, pp. 50-61. 1581 p

PUFFER, R.R. \& SERRANO, C.V.

1973 - Patterns of mortality in childhood. PAHO/WHO Scientific Publication, 262. Washington, D.C. 32 p.

WATERLOW, J.C. \& PAYNE, P.R.

1975 - The protein gap. Nature, 258:113-117.

WATERLOW, J.C. \& RUtishaUSER, I.H.E.

1974 - Eearly malnutrition and mental de velopment. Eds, L. Cravioto, L. Hambrauer and B. Vahlquist. Estocolmo.

Waterlow, J.C. \& Vergara, A.

1956 - Protein Malnutrition in Brazil. Food and Agriculture Organization, Rome.

WINICK, M. \& NOBLE, A.

1966 - Cellular responde in rats during malnutrition at various ages. J. Nutr. $89: 300-306$ 